\title{
Macrophages loaded CpG and GNR-PEI for combination of tumor photothermal therapy and immunotherapy
}

\author{
Jie Chen ${ }^{1 \dagger}$, Lin Lin $^{1 \dagger}$, Nan Yan ${ }^{1}$, Yingying $\mathrm{Hu}^{1}$, Huapan Fang ${ }^{1}$, Zhaopei Guo ${ }^{1}$, Pingjie Sun ${ }^{2}$, \\ Huayu $\operatorname{Tian}^{1^{*}}$ and Xuesi Chen ${ }^{1^{*}}$
}

\begin{abstract}
Nano-therapeutic approach for clinical implementation of tumors remains a longstanding challenge in the medical field. The main challenges are rapid clearance, offtarget effect and the limited role in the treatment of metastatic tumors. Toward this objective, a cell-mediated strategy by transporting photothermal reagents and $\mathrm{CpG}$ adjuvant within macrophage vehicles is performed. The photothermal reagents are constructed by conjugating of hyperbranched polyethyleimine (PEI) to golden nanorode (GNR) via S-Au bonds. GNR-PEI/CpG nanocomposites, formed via electrostatic interaction and displayed excellent near-infrared (NIR) photothermal performance, exhibit immense macrophage uptake and negligible cytotoxic effect, which is essential for the fabrication of GNR-PEI/CpG loaded macrophages. GNR-PEI/ CpG loaded macrophages demonstrated admirable photothermal response in vitro. Benefited from the functionalization of the binding adhesion between macrophages and 4T1 cells, GNR-PEI/CpG loaded macrophages significantly promoted tumor accumulation in vivo and dramatically enhanced the efficiency of photothermal cancer therapy. Moreover, the immune system is activated after photothermal therapy, which is mainly attributed to the generation of tumor specific antigens and CpG adjuvant in situ. Our findings provide a potential cell-mediated nanoplatform for tumor therapy by combination of near infrared photothermal therapy and immunotherapy.
\end{abstract}

Keywords: hyperbranched polymers, immunotherapy, macrophages, photothermal therapy, synergistic treatment

\section{INTRODUCTION}

Malignant tumor has evolved to be a serious threat to human health $[1,2]$. Although many strategies have been developed, permanent cures for tumor therapy have remained elusive [3]. To date, the main clinical therapeutic options are surgery, chemotherapy, radiotherapy and their combined treatments. However, there are still several bottleneck problems to be addressed, including multidrug resistance, undesirable side effects, heterogeneity, and poor permeability [4-7]. Recently, nanoparticle (NP)-mediated photothermal therapy (PTT) has attracted considerable attention due to thermal elimination of tumor cells by the localized conversion of near infrared (NIR) light into heat $[8,9]$. PTT reveals rapid, efficient, and minimally invasive capacities compared with other approaches. Multiple photothermal agents have been successful developed, such as melanin [10], indocyanine green [11], carbon nanotubes [12] and gold nanomaterials [13]. Particularly, gold nanorods (GNR) -based nanoparticles have been extensively investigated in biomedicine due to their unique shape effects $[8,14,15]$. GNR can produce stable photoacoustic signals that make them trackable in vivo. Photoacoustic signals can be reconstructed according to appropriate mathematical methods to obtain ultrasound images. Moreover, GNR can effectively absorb NIR light by its longitudinal surface plasmon resonance. The NIR light can deeply penetrate tissues without damaging normal tissues due to their weakly absorption by tissues and blood [16,17]. There-

\footnotetext{
${ }^{1}$ Key Laboratory of Polymer Ecomaterials, Changchun Institute of Applied Chemistry, Chinese Academy of Sciences, Changchun 130022, China

${ }^{2}$ Changchun Golden Transfer Science and Technology Co. Ltd., Changchun 130022, China

† These authors contributed equally to this work.

* Corresponding authors (emails: thy@ciac.ac.cn (Tian H); xschen@ciac.ac.cn (Chen X))
} 
fore, GNR is a promising agent for the combined advantages of photoacoustic imaging and photothermal therapy.

GNR has been utilized to construct nanocarriers for loading drugs/genes due to their optimal advantages of simple chemical modification on their surfaces. $\mathrm{Li}$ and coworkers constructed a gold nanorods-siRNA platform. The synergistic therapeutic effect against tumors was achieved by combining photothermal and gene therapy [18]. To improve the adaptability and targeting, Wang et al. $[19,20]$ developed a GNR-based gene delivery system, which consisted of GNR conjugated covalently with RGD (arginine-glycine-aspartic acid) peptides modified disulfide cross-linked short polyethyleimine (PEI). Significantly improved gene transfection efficiency was achieved by this novel system compared with commercial PEI, due to their capacities of targeting to tumor cells and escape from endo-lysosomal compartments. Although dramatic contributions to tumor therapy have been achieved by GNR based nanocarriers, as exogenous materials, they can be recognized and cleared immediately by immune systems once injected into the bloodstream, which severely restricts their clinical application [21-24].

Cell mediated strategy that can effectively circumvent the biological barriers has aroused extensive attention in the treatment of many kinds of tumors [25,26]. Among them, macrophages are attractive due to their innate phagocytotic capability. They are not only circulating cells in body which are easy to achieve, but also one of the most abundant cells in tumor microenvironments which are directly relevant to tumor proliferation and metastasis $[27,28]$. Macrophages can effectively bind to tumor cells via interactions between a 4 integrins and vascular cell adhesion molecule-1 (VCAM-1), thereby promoting their migration to tumor cells [29]. Macrophages can endocytose gold nanoparticles effectively and migrate into cancer cell spheroids without losing their photothermal performance [30]. Chu and coworkers prepared macrophages with GNR and improved the intratumoral distribution of GNR, thus enhancing the photothermal therapeutic efficacy [26,31]. Although these studies exhibited efficient migration and therapeutic capacity of GNR loaded macrophages, the therapeutic of metastatic tumor cells has not been demonstrated by this platform. Immunotherapy was considered as one of the most effective strategies to treat tumor metastasis [32]. Here, we hypothesized that photothermal therapy combined with immunotherapy may enhance the comprehensive antitumor effect by photothermal elimination and immune surveillance.

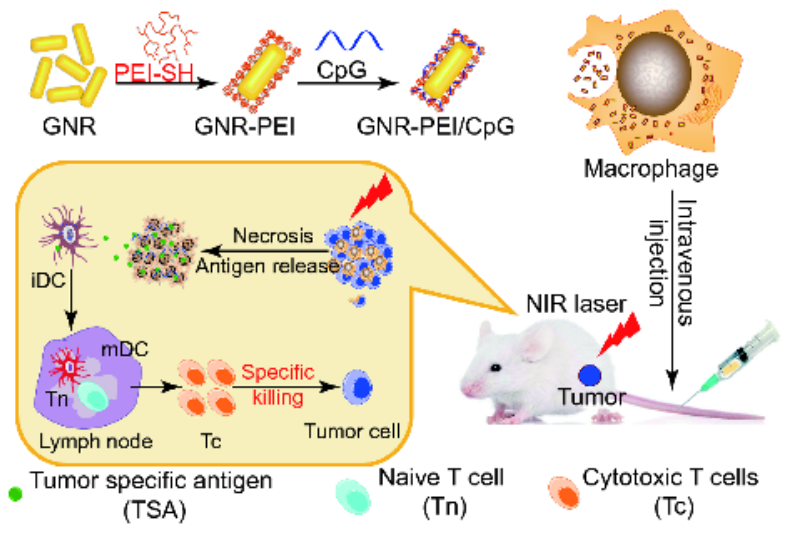

Scheme 1 Macrophages loaded GNR-PEI/CpG for tumor treatment by combination of photothermal therapy and immunotherapy.

Herein, hyperbranched PEI was conjugated onto the surface of GNR to construct a cationic nanocarrier, and then form nanocomplex with anionic immune adjuvant CpG by electrostatic interaction. Afterwards, we explored macrophages decorated GNR-PEI/CpG complex to circumvent biological barriers and improve specific targeting capability (Scheme 1). We first encapsulated photothermal reagents and adjuvant simultaneously into macrophages to generate GNR-PEI/CpG-laden-macrophages for tumor targeting therapy. The photothermal ablation-induced tumor lysis could liberate tumor specific antigens. GNR-PEI/CpG complexes in tumor further improved the internalization of $\mathrm{CpG}$ by plasmacytoid dendritic cells. The strong photothermal effect appeared after intravenous injection of GNR-PEI/CpG-laden macrophages under NIR light irradiation, thus conducing to the photothermal elimination of tumors and minimizing the metastasis and recurrence.

\section{EXPERIMENTAL SECTION}

Tetrachloroauric acid $\left(\mathrm{HAuCl}_{4} \cdot 4 \mathrm{H}_{2} \mathrm{O}\right)$ was purchased from Shanghai Chemical Reagent Co. Ltd. (Shanghai, China). Sodium borohydride $\left(\mathrm{NaBH}_{4}, 99 \%\right)$ was purchased from Tianjin FuChen Chemical Reagents Factory (Tianjin, China). Cetyltrimethylammonium bromide (CTAB, 99\%) and $\mathrm{N}$-hydroxysuccinimide (NHS, 98\%) were purchased from Aladdin (Shanghai, China). 1-Ethyl3-(3-(dimethylamino)propyl)carbodiimide (EDC. $\mathrm{HCl}$ ) was purchased from GL Biochem Ltd. (Shanghai, China). Branched polyethylenimine with average molecular weight of $25 \mathrm{kDa}$ (PEI25k, 99\%) was purchased from Aldrich. 3-Mercaptopropionic acid (MPA, 99\%) was purchased from Alfa Aesar. 3-(4,5-Dimethyl-2-thiazolyl)2,5-diphenyltetrazolium bromide (MTT) was purchased 
from Amresco (Solon, OH, USA). Dimethyl sulfoxide (DMSO) was purchased from Tianjin Yongda Chemical Reagent Co. Ltd. (Tianjin, China). Dulbecco's modified Eagle's medium (DMEM) was purchased from Gibco (Grand Island, NY, USA). Single-stranded CpG ODN 1826 (5'-TCCATGACGTTCCTGACGTT-3') was purchased from Sangon Biotech (Shanghai, China) Co., Ltd. All the antibodies were purchased from ebioscience (USA). Deionized water was used throughout the experiments. Other reagents and solvents were purchased from Sinopharm Chemical Reagent Co. Ltd. without further treatment.

The GNRs were prepared using a seed-mediated growth strategy as described previously $[8,33,34]$. Briefly, $500 \mu \mathrm{L}$ of $0.01 \mathrm{~mol} \mathrm{~L}^{-1} \mathrm{HAuCl}_{4}$ was mixed with $20 \mathrm{~mL}$ of $0.1 \mathrm{~mol} \mathrm{~L}^{-1} \mathrm{CTAB}$ solution, and then $600 \mu \mathrm{L}$ of freshly prepared $0.01 \mathrm{~mol} \mathrm{~L}^{-1}$ ice-cold $\mathrm{NaBH}_{4}$ was added immediately under stirring. The seed solution was allowed to keep for $2 \mathrm{~h}$ to form CTAB-capped gold nanoparticles. Afterwards, $200 \mathrm{~mL}$ of $0.1 \mathrm{~mol} \mathrm{~L}^{-1} \mathrm{CTAB}$ and $2 \mathrm{~mL}$ of $0.01 \mathrm{~mol} \mathrm{~L}^{-1} \mathrm{AgNO}_{3}$ was added to $10 \mathrm{~mL}$ of $0.01 \mathrm{~mol} \mathrm{~L}^{-1}$ $\mathrm{HAuCl}_{4}$ solution in a flask, and then $2 \mathrm{~mL}$ of $1 \mathrm{~mol} \mathrm{~L}^{-1}$ $\mathrm{HCl}$ and $1.4 \mathrm{~mL}$ of $0.1 \mathrm{~mol} \mathrm{~L}^{-1}$ ascorbic acid was added under stirring until the color changed into colorless. Furthermore, $200 \mu \mathrm{L}$ of the seed solution was added and left undisturbed overnight. Finally, GNRs were collected by centrifugation for $15 \mathrm{~min}$ at $10,000 \mathrm{rpm}$ and washed twice with ultrapure water.

GNR-PEI was synthesized by bonding hyperbranched PEI on the surface of GNRs via sulfur-gold bond [8]. Briefly, $0.2 \mathrm{~g}$ of $\mathrm{N}$-hydroxysuccinimide, $0.2 \mathrm{~g}$ of ethylene dichloride, and $2 \mathrm{~g}$ of PEI were added to $60 \mathrm{~mL}$ of aqueous solution containing $0.02 \mathrm{~g}$ of methiopropamine for $24 \mathrm{~h}$ at $30^{\circ} \mathrm{C}$. Then, $10 \mathrm{mg}$ of GNRs was mixed with thiolated PEI solution and reacted for another $24 \mathrm{~h}$ under stirring. Finally, GNR-PEI was separated via centrifugation at 10,000 rpm for $10 \mathrm{~min}$, washed twice, and redispersed in deionized water. The morphology was observed by transmission electron microscopy (TEM). Briefly, the samples $\left(0.2 \mathrm{mg} \mathrm{mL}^{-1}\right)$ were dropped onto a 200 -mesh copper grid coated with carbon and dried at room temperature. ${ }^{1} \mathrm{H}$ NMR spectra were recorded in $\mathrm{D}_{2} \mathrm{O}$ at $25^{\circ} \mathrm{C}$. TEM measurements were carried out on a JEOL JEM1011 electron microscope at an acceleration voltage of $100 \mathrm{kV}$. The UV-vis-NIR absorption spectra were measured on a multifunction microplate scanner (Tecan Infinite M 200). The contents of gold and PEI in the product were measured by thermal gravimetric analysis (TGA).

Mouse macrophages (RAW264.7 cells), breast tumor cells (4T1 cells), and mouse skin fibroblast cells (L929 cells) were cultured in the DMEM culture medium consisting of $10 \%$ heat-inactivated fetal bovine serum, 100 units $\mathrm{mL}^{-1}$ penicillin, and $63 \mathrm{mg} \mathrm{mL}^{-1}$ streptomycin and kept in a humidified atmosphere of $5 \% \mathrm{CO}_{2}$ at $37^{\circ} \mathrm{C}$.

The cytotoxicity of GNR-PEI and GNR-PEI/CpG was evaluated by MTT assay in RAW264.7 cells. Briefly, the cells were seeded at a density of $1.0 \times 10^{4}$ cells per well in a 96-well plate and incubated for $24 \mathrm{~h}$ before treating with various samples at the different concentrations. The final concentration of CpG was $1 \mu \mathrm{g} \mathrm{mL}^{-1}$. After incubation for $24 \mathrm{~h}, 20 \mathrm{~mL}$ of MTT stock solution $\left(5 \mathrm{mg} \mathrm{mL}^{-1}\right.$ in PBS) was added. After $4 \mathrm{~h}$, the medium was carefully removed and $150 \mu \mathrm{L}$ of DMSO was added to dissolve the MTT formazan crystals. Afterwards, the absorbance $(492 \mathrm{~nm})$ was evaluated using an ELISA microplate reader (BioRad). The cell viability was normalized to that of the cells cultured only in DMEM medium.

To verify intracellular translocation of GNR-PEI/CpG, RAW264.7 cells were cultured on a 24-well plate at $2.0 \times 10^{5}$ cells per well for $24 \mathrm{~h}$, and then incubated with GNR-PEI/CpG (the optimal concentration filtrated by MTT assay) at various time points (1-9 h) CpG was labeled by carboxyfluorescein (FAM) and the final concentration was $1.0 \mu \mathrm{g} \mathrm{mL}^{-1}$ in all groups. After harvesting, the cells were washed and resuspended with cold PBS. Finally, the intracellular uptake was performed using a Guava easyCyte 6-2L Base System (Merck Millipore, USA). The efficiency of endocytosis (\%) was calculated automatically by flow cytometry according to the following equation: Endocytosis $(\%)=\left(A_{\text {endocytosis }} / A_{\text {all }}\right) \times 100$, where $A_{\text {endocytosis }}$ is the cell numbers that endocytosed FAM-CpG and $A_{\text {all }}$ is the cell numbers of all the tested cells. For CLSM assay, RAW264.7 cells $\left(3.0 \times 10^{5}\right.$ cells per well) were seeded in a 6-well plate with a coverslip in each well for $24 \mathrm{~h}$. The cells were incubated with GNR-PEI/ CpG for $5 \mathrm{~h}$, and fixed in paraformaldehyde (PFA, $4 \%$ in PBS) for $15 \mathrm{~min}$ at room temperature. Afterwards, the coverslips were rinsed with cold PBS for three times, and then the cell nuclei were stained with DAPI for $3 \mathrm{~min}$. Subsequently, the coverslips were washed thrice and enclosed with glycerol. Finally, the coverslips were visualized using confocal laser scanning microscope (CLSM, LSM780, Carl Zeiss Inc., Germany).

RAW264.7 cells $\left(1.0 \times 10^{4}\right.$ cells per well $)$ were seeded in a 96-well plate for $24 \mathrm{~h}$. Afterwards, GNR-PEI/CpG (20:1, $w / w)$ were added into each well. The final concentration of CpG was $1 \mu \mathrm{g} \mathrm{mL}^{-1}$. NIR laser $\left(808 \mathrm{~nm}, 1 \mathrm{~W} \mathrm{~cm}^{-2}\right)$ was performed to irradiate each well for $10 \mathrm{~min}$ after incubation for $5 \mathrm{~h}$ and replacement of the fresh medium. 
The cells without any treatment were set as the control group. After incubation for another $5 \mathrm{~h}$, the cell apoptosis assay was carried out by an annexin V-FITC and PI apoptosis detection kit according to the manufacturer's instructions.

Live-dead cell staining assay was further performed after NIR laser irradiating. Briefly, the cells were stained with Calcein-AM $\left(2 \mu \mathrm{mol} \mathrm{L} \mathrm{L}^{-1}\right)$ and propidium iodide $\left(4 \mu \mathrm{mol} \mathrm{L}^{-1}\right)$ for $30 \mathrm{~min}$ in the dark at room temperature. Finally, the fluorescent photographs were detected by a fluorescence microscope in the dark (Nikon TI-S, Japan).

To verify how many GNR-PEI/CpG-laden-macrophages can trigger the photothermal conversion in vitro, we cultured RAW264.7 cells on a $90 \mathrm{~mm}$ dish. Then, GNR-PEI/CpG was added into the dish and incubated for $5 \mathrm{~h}$. Next, the medium was removed and the cells were washed carefully with cold PBS. The GNR-PEI/CpG-laden-macrophages harvested after digesting by trypsin. Finally, the cells were counted, divided into several groups, and irradiated by NIR laser. The temperature of the samples was monitored every $10 \mathrm{~s}$.

The migration activities of macrophages were measured by a transwell assay. $100 \mu \mathrm{L}$ of RAW264.7 cells $\left(1.0 \times 10^{5}\right.$ cells) were seeded to the upper chambers of inserts (pore size, $8.0 \mu \mathrm{m})$. Then, $500 \mu \mathrm{L}$ of DMEM media with $1.0 \times 10^{5}$ of $4 \mathrm{~T} 1$ cells or L929 cells were respectively added to the lower chambers of 24-well plate. DMEM media without any treatment were used as a negative control. After $24 \mathrm{~h}$ of incubation, the macrophages not migrating to the bottom side were gently swept off by cotton swab, while the migrated macrophages cells across the transwells were stained with violet crystal and photographed.

RAW264.7 cells were incubated with GNR-PEI/CpG for $5 \mathrm{~h}$ and labeled with CellTrace CFSE Cell Proliferation Kit according to the manufacturer's instructions. 4T1 cells and L929 cells were pre-labeled with DiR. Afterwards, RAW264.7 cells and 4T1 cells were co-cultured in a 6-well plate. L929 cells were used as the negative control. After incubation for $24 \mathrm{~h}$, the cells were washed for three times with cold PBS and the fluorescence images were acquired on the Nikon TI-S microscope.

To obtain extracellular secretions (ES), different cells (L929 cells, RAW264.7 cells, and GNR-PEI/CpG-ladenmacrophages) were cultured on $90 \mathrm{~mm}$-plates within $10 \mathrm{~mL}$ of DMEM medium. After incubation for $24 \mathrm{~h}$, ES were harvested and filtrated through $0.22 \mu \mathrm{m}$ syringe filters to filter out cellular debris. ES induced cytotoxicity assay on 4T1 cells were evaluated by MTT assay as described previously. Briefly, the media were replaced with ES after $24 \mathrm{~h}$ incubation of $4 \mathrm{~T} 1$ cells in 96-well plates.
Then, the cells were incubated with: 100\% ES, 50\% DMEM+50\% ES, 75\% DMEM+25\% ES, 87.5\% DMEM $+12.5 \%$ ES, $93.75 \%$ DMEM+6.25\% ES, and 100\% DMEM. After incubation for $24 \mathrm{~h}, 4 \mathrm{~T} 1$ cells were harvested and measured by MTT assay.

The photothermal therapy of GNR-PEI/CpG-ladenmacrophages was further verified in vivo. The female $\mathrm{Balb} / \mathrm{c}$ mice (6-8 weeks old, 18-20 g) were purchased and kept under the guidance of the Animal Care and Use Committee of Jilin University. $2.0 \times 10^{6}$ of $4 \mathrm{~T} 1$ cells in $200 \mu \mathrm{L}$ PBS was subcutaneously injected into the right flank of mice. When the average diameter of the tumors reached about $6 \mathrm{~mm}$, a large amount of GNR-PEI/CpGladen-macrophages were harvested and injected by intravenous injection at $2.0 \times 10^{6}$ cells per mouse. After $24 \mathrm{~h}$, a NIR laser $(808 \mathrm{~nm}, 1 \mathrm{~W} \mathrm{~cm}$ ) was performed to irradiate the tumors for $10 \mathrm{~min}$. Simultaneously, the temperature variation of tumor sites was monitored and thermographic maps were also obtained by using an infrared thermal camera. PBS group was used as the negative control. After $72 \mathrm{~h}$, the mice were executed, the tumors were extracted to evaluate the recruitment and maturation of DC cells $\left(\mathrm{CD} 11 \mathrm{c}^{+} \mathrm{CD} 86^{+}\right.$, ebioscience, USA), the popliteal lymph nodes were cut out to further confirm the maturation of DC cells, and the harvested splenocytes from mice were used to monitor the amount of $\mathrm{T}$ cells $\left(\mathrm{CD}^{+} \mathrm{CD}^{+}\right.$and $\mathrm{CD} 3^{+} \mathrm{CD} 8^{+}$, ebioscience, USA).

Statistical analysis was performed using Student's $t$-test. All the data were given by a mean value together with its standard deviation (mean $\pm \mathrm{SD}$ ). If $P<0.05$, the differences were regarded to be significant, while for $P<0.01$, it could be regarded as extremely significant.

\section{RESULTS AND DISCUSSION}

GNR were prepared according to the seed-mediated growth method [34]. Sulfydryl groups were introduced onto the surface of hyperbranched PEI. The affiliation of peaks on PEI-SH was shown in the ${ }^{1} \mathrm{H}$ NMR spectra and the characteristic peaks of sulfydryl groups on PEI-SH were at 2.99 and 3.14. The molar content of sulfydryl segment in PEI-SH was about 23.3 (Fig. S1), which could be calculated from peak intensities of proton signal of sulfydryl and PEI. GNR-PEI was then constructed by conjugating of PEI-SH to GNR via S-Au bonds (Fig. 1). The geometric morphology of GNR-PEI was achieved by TEM and the images showed that GNR-PEI had a mean length of $91.5 \pm 8.2 \mathrm{~nm}$ and width of $30.4 \pm 2.5 \mathrm{~nm}$ (Fig. 2a). There was little effect on the morphology of GNR-PEI/CpG complex compared with GNR-PEI (Fig. 2b). As shown in Fig. 2c, a maximum absorption 


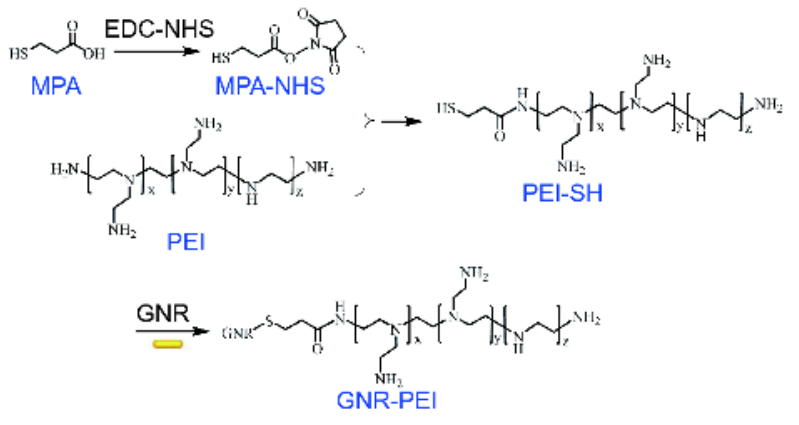

Figure 1 Synthesis route of GNR-PEI.
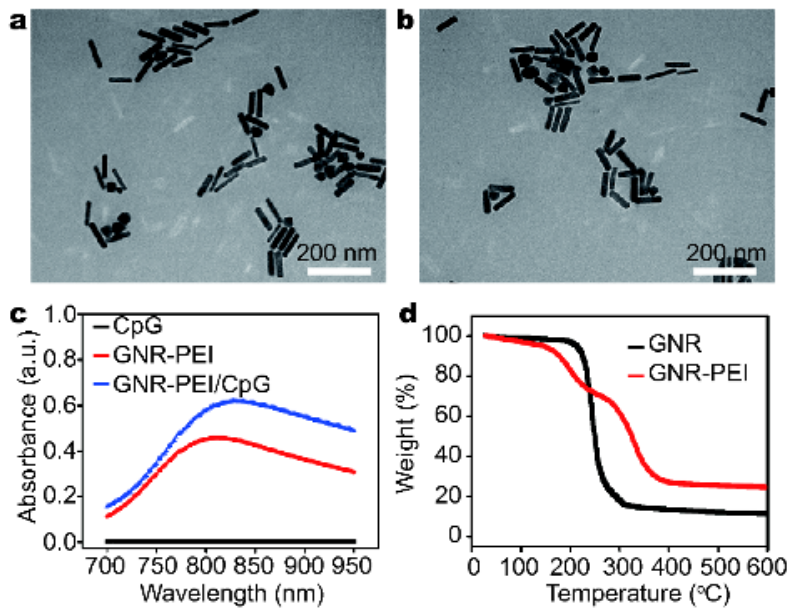

Figure 2 The geometric morphology and absorption spectra of GNRPEI and GNR-PEI/CpG. TEM images of (a) GNR-PEI and (b) GNR$\mathrm{PEI} / \mathrm{CpG}$ (scale bar $=200 \mathrm{~nm}$ ), (c) UV-vis absorption spectra of GNRPEI and GNR-PEI/CpG (the final concentration of GNR-PEI was $20 \mu \mathrm{g} \mathrm{mL}^{-1}$ ), (d) TGA curves of GNR and GNR-PEI.

peak for the original GNR-PEI was around $802 \mathrm{~nm}$. While for GNR-PEI/CpG complex, the peak slightly redshifted to $817 \mathrm{~nm}$ and the intensity also exhibited obvious enhancement, which was mainly due to the increased dielectric constant and the formation of hydrogen bonding between GNR-PEI and CpG [35]. For naked CpG group, no absorption was detected under our experimental conditions. The results indicated that GNRPEI had the potential capacity for photothermal conversion. The content of PEI-SH on the surface of GNR was determined by TGA. The measurements were carried out from room temperature to $600^{\circ} \mathrm{C}$ at a heating rate of $10^{\circ} \mathrm{C}$ $\min ^{-1}$ in $\mathrm{N}_{2}$. The results (Fig. 2c) showed that the content of PEI-SH in GNR-PEI was about 34.3\%.

To determine the optimum dosage of GNR-PEI/CpG, the cytocompatibility was evaluated by MTT assay in RAW264.7 cells. The cell viability of untreated cells was

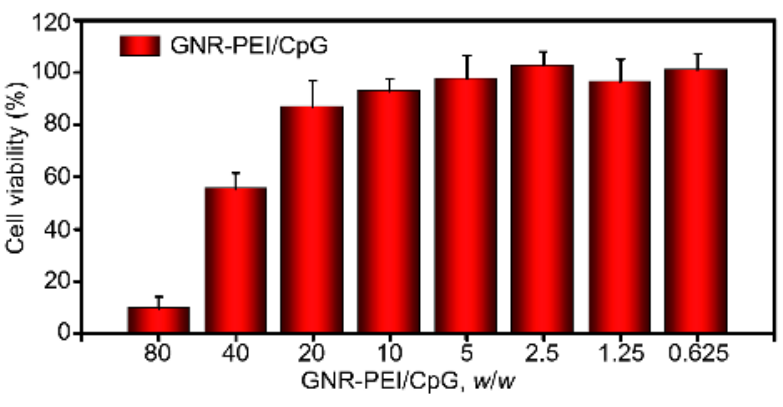

Figure 3 Cytocompatibility of GNR-PEI/CpG in RAW264.7 cells.

regarded as $100 \%$. As shown in Fig. 3, the cell viability was more than $85 \%$ when the weight ratio of GNR-PEI/ CpG was 20:1. Therefore, this optimal ratio was chosen for the following studies. In addition, the cell viability of GNR-PEI itself was similar to that of GNR-PEI/CpG (Fig. S2).

To screen out the incubation time of the maximum amount of endocytosis, the intracellular efficiency was evaluated by flow cytometry (FCM). FCM analysis (Fig. 4a) showed that the endocytosis increased incrementally over time initially. The peak appeared in the $4-6 \mathrm{~h}$ after internalization. Therefore, $5 \mathrm{~h}$ of endocytosis was used in the following studies. The intracellular uptake of GNR-PEI/CpG complex was further evaluated by FCM after incubating for $5 \mathrm{~h}$ (Fig. 4b). The fluorescence peaks for GNR-PEI/CpG complex shifted to right remarkably, indicating the significant internalization efficiency that was comparable with the commercial reagent PEI25k. Furthermore, the intracellular uptake of GNR-PEI/CpG was monitored by CLSM. As shown in Fig. 4c, the high fluorescent signals in RAW264.7 cells treated with GNR$\mathrm{PEI} / \mathrm{CpG}$ complex were prominent and similar to that of PEI25k/CpG complex, which was consistent with the former FCM analysis. These results might provide a powerful theoretical basis for the macrophages therapy of tumors.

The photothermal performance of GNR-PEI/CpG-laden-RAW264.7 cells in PBS was investigated with different laser powder density and various cell numbers. The photothermal performance of GNR-PEI/CpG-ladenRAW264.7 cells at $2.5 \times 10^{6}$ cells $\mathrm{mL}^{-1}$ with different power densities ( $808 \mathrm{~nm}, 1.0$ or $2.0 \mathrm{~W} \mathrm{~cm}^{-2}, 10 \mathrm{~min}$ ) was carried out. The temperature was too low to achieve the subsequent treatment with the power density of $1.0 \mathrm{~W} \mathrm{~cm}{ }^{-2}$ $\left(32.5^{\circ} \mathrm{C}\right)$, while for $2.0 \mathrm{~W} \mathrm{~cm}{ }^{-2}$, the peak temperature could only reach to $44^{\circ} \mathrm{C}$. For one thing, this temperature is restricted to induce effective phtotothermal therapy, for another, the laser intensity is too high to be used in vivo. 

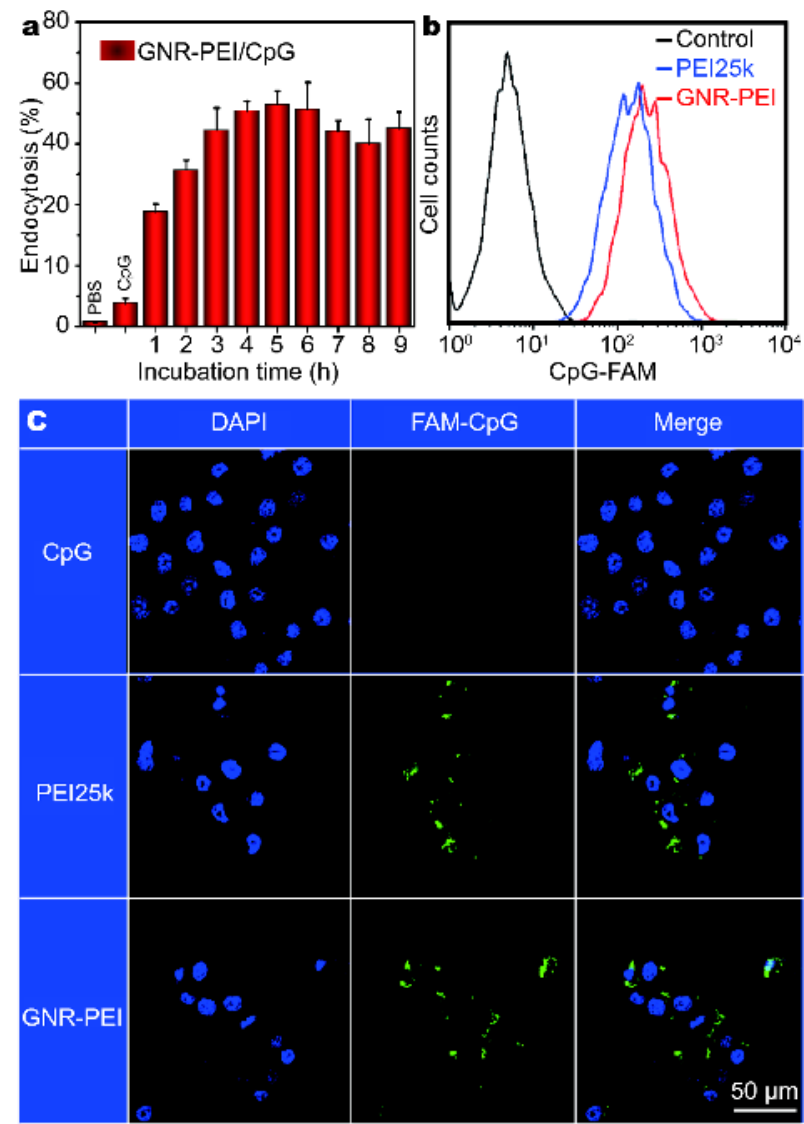

Figure 4 Cellular uptake of GNR-PEI/CpG in RAW264.7 cells. (a) Flow cytometry analysis of cellular uptake of GNR-PEI/CpG at different incubation time. (b) Cellular uptake of GNR-PEI/CpG for $5 \mathrm{~h}$, the commercial PEI25k was used as the control. (c) CLSM observation of the cellular uptake of GNR-PEI/CpG and PEI25k/CpG for $5 \mathrm{~h}$. The final concentration of GNR-PEI was $20 \mu \mathrm{g} \mathrm{mL}^{-1}$. CpG was labeled with carboxyfluorescein (FAM, green) and the nucleus was labeled with DAPI (blue). Scale bar is $50 \mu \mathrm{m}$.

Afterwards, we evaluated the effect of cell numbers on photothermal conversion capability. As shown in Fig. 5a, with the increasement of cell numbers from $0.5 \times 10^{6}$ to $2.5 \times 10^{6}$ cells $\mathrm{mL}^{-1}\left(808 \mathrm{~nm}, 1.0 \mathrm{~W} \mathrm{~cm} \mathrm{~cm}^{2}, 10 \mathrm{~min}\right)$, the maximum temperature reached from 32.5 to $51.6^{\circ} \mathrm{C}$, which was high enough to cause hyperthermia in macrophages.

The photothermal toxicity of GNR-PEI/CpG-ladenRAW264.7 cells was evaluated by calcein AM/PI live/dead cell staining and flow cytometry. Fluorescence staining of living/dead cells proved the excellent photothermal efficacy of GNR-PEI/CpG-laden-RAW264.7 cells with Laser, while only a few dead cells with red fluorescence were observed for GNR-PEI/CpG-laden-RAW264.7 cells group (Fig. 5b). The apoptosis of macrophages was analyzed by

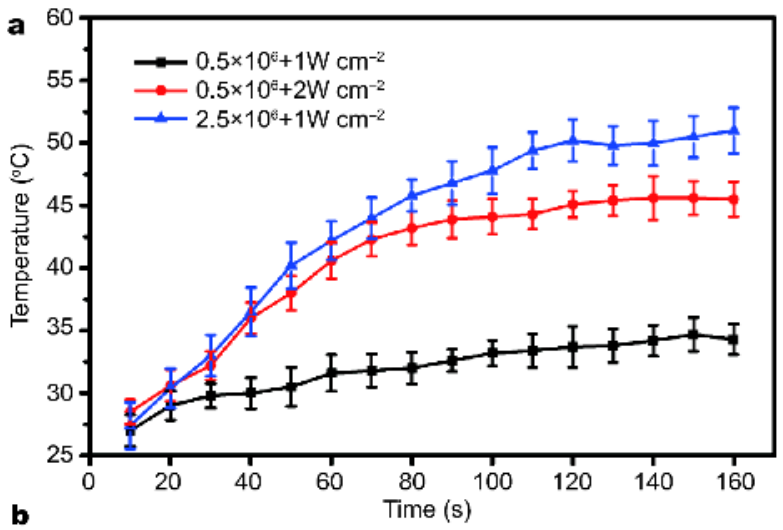

b
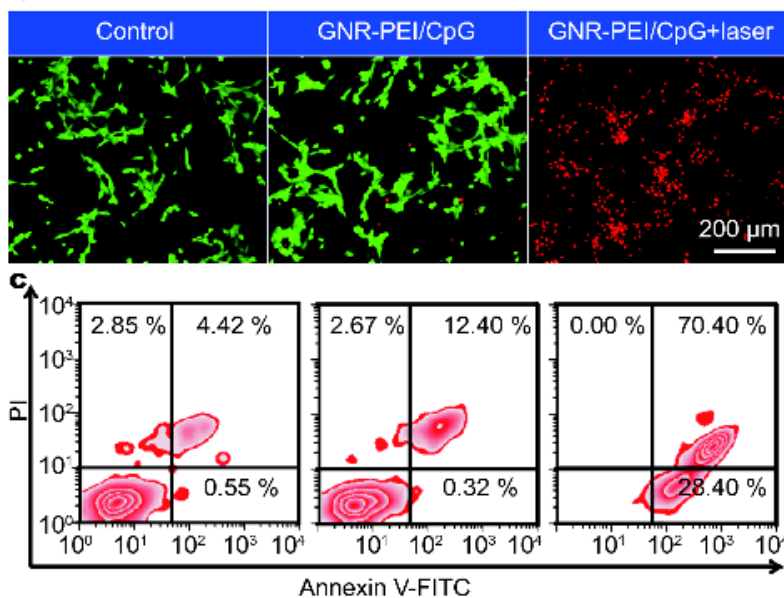

Figure 5 Photothermal effect of GNR-PEI/CpG-laden-RAW264.7 cells. (a) RAW264.7 cells were incubated with GNR-PEI/CpG for $5 \mathrm{~h}$ and the thermographic monitoring was measured in the conditions of different cell numbers and laser intensity. (b) Calcein AM/PI staining (live cells were stained green with Calcein AM and dead cells were stained red with PI), the scale bar is $200 \mu \mathrm{m}$. (c) Cell apoptosis analysis (apoptotic cells were stained with Annexin V-FITC and dead cells were stained with PI).

FCM. Fig. $5 c$ revealed that GNR-PEI/CpG's treatment could inconsiderably increase the proportion of apoptotic cells compared with the control group. For GNR-PEI/ CpG-laden-RAW264.7 cells irradiated by laser, the proportions of early apoptotic cells and late apoptotic cells were significantly increased to $28.4 \%$ and $70.4 \%$, respectively. Evidently, GNR-PEI/CpG complexes were desirable in macrophages delivery due to their low cytotoxicity and could trigger efficient apoptosis under NIR laser irradiation.

To explore the potential application of GNR-PEI/CpGladen-macrophages in vivo, we initially tested the effect of cultured macrophages supernatant on the proliferation of $4 \mathrm{~T} 1$ cells. As shown in Fig. 6, the cytotoxic results showed that $25 \%$ of EM dilution medium could not reduce the 


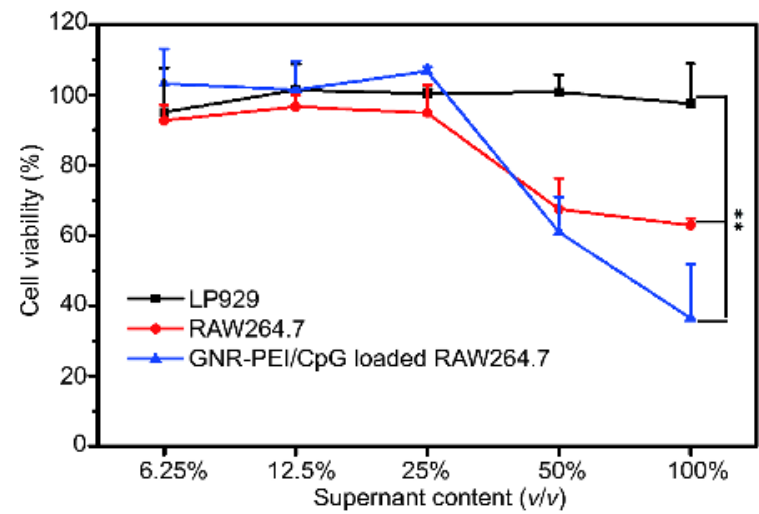

Figure 6 The effects of conditioned extracellular secretions (ES) on 4T1 tumor cells. Data represent mean $\pm \mathrm{SD}$ with four replicates. ${ }^{\star *} P<0.01$.

proliferation of $4 \mathrm{~T} 1$ cells, while $50 \%$ and $100 \%$ significantly reduced the proliferation of $4 \mathrm{~T} 1$ cells. Furthermore, $100 \%$ of EM dilution medium of GNR-PEI/ CpG-laden macrophages exhibited more remarkable inhibition of tumor cells compared with pure macrophages. In addition, no significant effect of L929 fibroblasts conditioned medium was found on 4T1 cancer cells (Fig. 6). Inspired by the above results, some of the cytokines secreted from macrophages might act a key role in inhibiting the proliferation of $4 \mathrm{~T} 1$ cells, but what these factors are and how they manipulate still need further investigated.

To determine tumor cells induced migration of macrophages, we performed a transwell assay. In this study, RAW264.7 cells in the upper chambers of inserts were exposed in the conditions of 4T1 cells, L929 cells and DMEM media, and the migrated cells across the chambers were monitored. The in vitro transwell migration assays showed that the motility of macrophages significantly improved in the condition of 4T1 tumor cells compared with L929 fibroblast cells (Fig. 7a), mainly attributed to the affinity between macrophages and 4T1 tumor cells. The affinity of macrophages and 4T1 cells was further conducted by co-culture experiments. The fluorescence images in Fig. $7 \mathrm{~b}$ indicated that more colocalization cells (white circles) were observed in the coculture group of macrophages and 4T1 cells. This further intuitively verified the former conclusion in transwell assay and provided strong evidence for cell therapy in vivo.

The in vivo photothermal effects were performed in $4 \mathrm{~T} 1$ xenograft tumor bearing Balb/c mice. Under NIR laser irradiation, the elevation of temperature could be detected directly, indicating the photothermal activity of

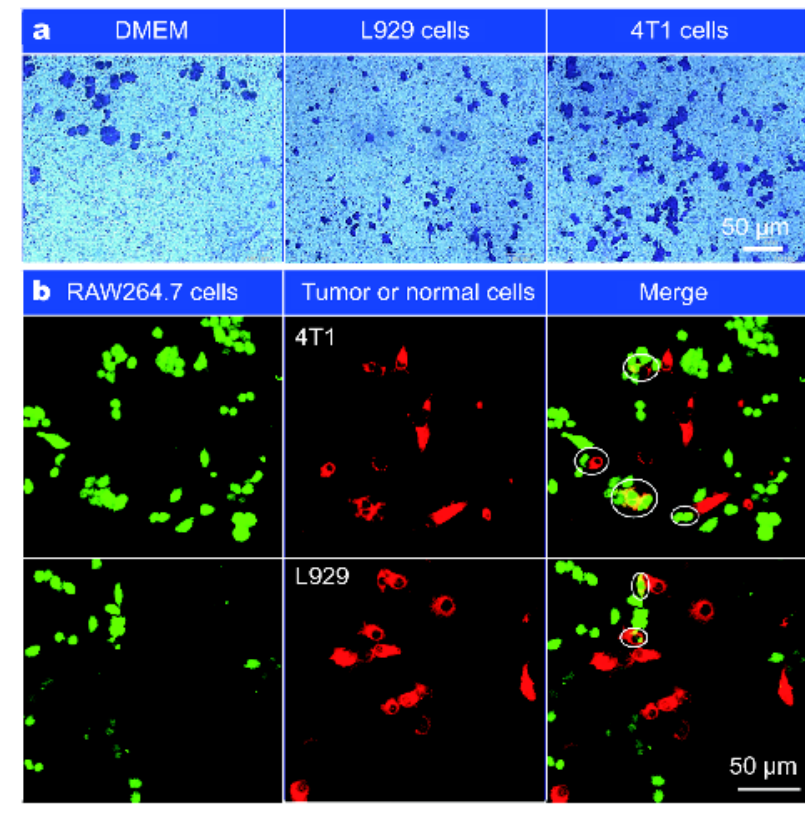

Figure 7 The in vitro migration effect of RAW264.7 cells. (a) Migration assays were performed using transwell chambers, (b) tumor cell tropism assay of macrophages. Macrophages were labeled with CFSE (green) and other cells were labeled with DIR (red). The overlapping cells were marked in circles (white circles). The scale bar is $50 \mu \mathrm{m}$.

GNR-PEI/CpG-laden macrophages was still kept in vivo after intravenous injection. For tumor-bearing mice injected with GNR-PEI/CpG-laden macrophages and PBS, the local tumor temperature could reach 51.7 and $39.8^{\circ} \mathrm{C}$, respectively (Fig. 8a, b). Furthermore, the temperature change in the tumor site was obtained using the infrared thermal camera during irradiation (Fig. 8c). The strong photothermal effect appeared after intravenous injection of GNR-PEI/CpG-laden-macrophages under NIR light irradiation, thus conducing to the photothermal elimination of tumors and minimizing the metastasis and recurrence. Shen et al. [36] demonstrated that GNRs modified with cationic carrier achieved both transfection performance and photothermal effect. Whereas, the risk brought from PEI25k mainly restricted its further application. In our previous study, we constructed GNRsPEI1.8k to achieve three functions in one work including high gene transfection efficiency, photoacoustic imaging and photothermal therapy [8]. Although the comprehensive performance of GNR-PEI was excellent, systemic in vivo treatment was still limited. In this study, we first encapsulated GNR-PEI and CpG adjuvant simultaneously into macrophages to generate GNR-PEI/CpG-ladenmacrophages for tumor targeting therapy. Combined with the former data, GNRs-PEI1.8k with its excellent multi-functions could further validate its potential ap- 

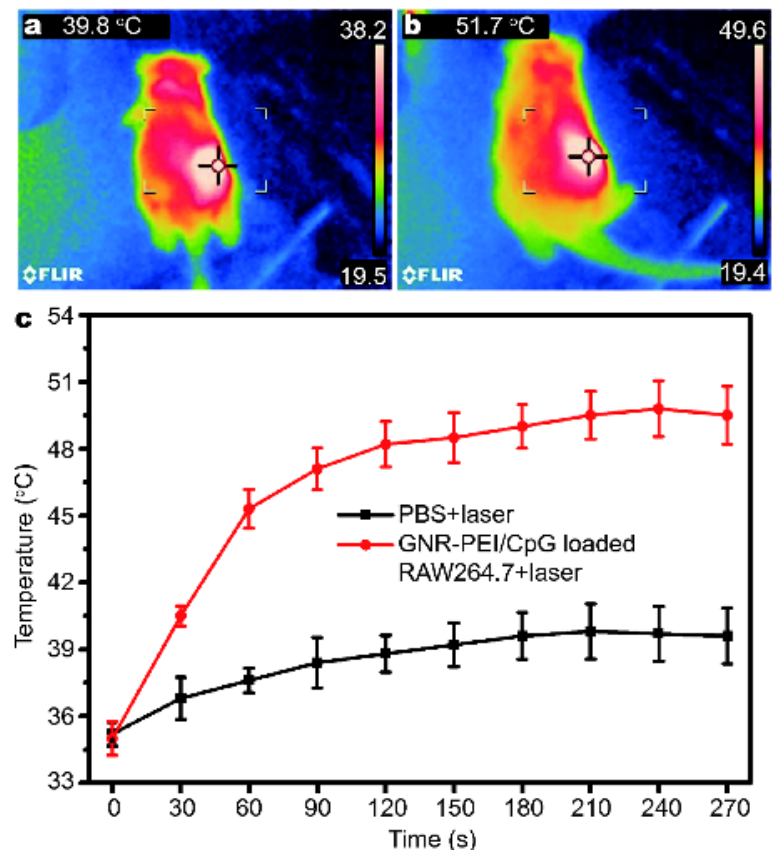

Figure 8 In vivo photothermal effect of GNR-PEI/CpG-laden-macrophages. The $4 \mathrm{~T} 1$ tumor-bearing Balb/c mice were intravenous injected with PBS and GNR-PEI/CpG-laden-macrophages. Afterwards, the thermographic images and temperature change of tumor sites were measured after NIR irradiation $\left(1.0 \mathrm{~W} \mathrm{~cm}^{-2}, 10 \mathrm{~min}\right)$. The near-infrared thermographic images were captured after $240 \mathrm{~s}$ of tumor-bearing mice after NIR irradiation: (a) PBS, (b) GNR-PEI/CpG-laden-macrophages. (c) Thermographic monitoring in the tumor sites of PBS and GNR-PEI/ CpG-laden-macrophages treated tumor-bearing mice.

plication in diagnosis and treatment of tumors.

Photothermal ablation-induced tumor lysis could liberate tumor specific antigens and $\mathrm{CpG}$ adjuvant in situ. Plasmacytoid dendritic cells were then recruited and activated through activation of toll-like receptor 9 signaling, thus initiating effective and systemic antitumor immunity through a complicated immune cascade. Previous studies showed that $\mathrm{CpG}$ could effectively activate dendritic cells and elicit adaptive immune responses in vivo [37]. Here, histological analysis of 4T1 tumors following intravenous administration of GNR-PEI/CpG-laden-macrophages plus laser treatment revealed a dramatic infiltration of dendritic cells (CD11 $\mathrm{c}^{+}, 14.78 \%$ ) in tumor sites compared with PBS (3.49\%) and GNR-PEI/CpG-laden-macrophages without laser group (10.23\%). The proportion of mature dendritic cells $\left(\mathrm{CD} 11 \mathrm{c}^{+} \mathrm{CD} 86^{+}\right)$was 8 -fold higher after photothermal therapy compared with saline-treated group (Fig. 9a). In addition, the treatment with GNRPEI/CpG-laden-macrophages with laser induced much more mature myeloid dendritic cells migrating to tumordraining lymph nodes compared with other groups. Fi-

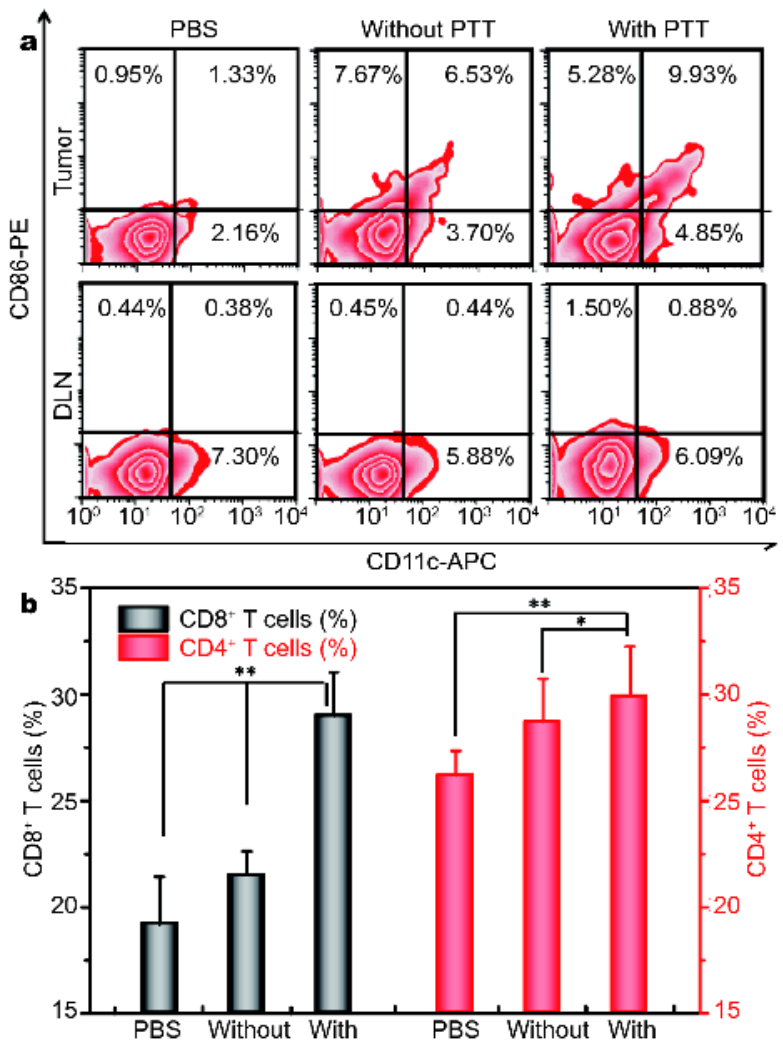

Figure $94 \mathrm{~T} 1$ tumor-bearing mice were given intravenous injections of PBS and GNR-PEI/CpG-laden macrophages at $2.5 \times 10^{6}$ cells per mouse with or without laser treatment $\left(1.0 \mathrm{~W} \mathrm{~cm}^{-2}, 10 \mathrm{~min}\right)$. (a) Flow cytometric analysis of activated myeloid dendritic cells in tumors or draining lymph nodes, (b) the proportion of $\mathrm{CD}^{+} \mathrm{T}$ cells (gray) and $\mathrm{CD} 4^{+} \mathrm{T}$ cells (red) in spleens after various treatments. Data represent mean $\pm \mathrm{SD}$ with four replicates. ${ }^{\star *} P<0.01,{ }^{\star} P<0.5$.

nally, the proportion of T-lymphocytes was further evaluated in spleens, and both $\mathrm{CD}^{+} \mathrm{T}$ cells and $\mathrm{CD}^{+} \mathrm{T}$ cells were significantly increased compared with salinetreated group (Fig. 9b). Collectively, these results indicated that photothermal ablation and the photothermally triggered tumor specific antigens and $\mathrm{CpG}$ adjuvant has a combined effect on the activation of myeloid dendritic cells following intravenous injection with GNR-PEI/CpG-laden-macrophages plus laser treatment. Our findings provided a cell-mediated potential nanoplatform for tumor therapy by combination of near infrared photothermal therapy and immunotherapy.

\section{CONCLUSIONS}

In this study, we described a cell-mediated strategy by transporting photothermal reagent (GNR-PEI) and CpG adjuvant within macrophage vehicles. GNR-PEI/CPG nanocomposites exhibited immense macrophage uptake 
and negligible cytotoxic effect in RAW264.7 cells. GNRPEI/CpG-laden-macrophages demonstrated admirable photothermal response in vitro. GNR-PEI/CpG-ladenmacrophages significantly promoted tumor accumulation in vivo and dramatically enhanced the efficiency of photothermal cancer therapy. The macrophages-laden-GNR$\mathrm{PEI} / \mathrm{CpG}$ nanocomplexes could promote CpG uptake by plasmacytoid dendritic cells after photothermal therapy. Moreover, the immune system is further activated, which is mainly attributed to the generation of tumor specific antigens and CpG adjuvant in situ. We first encapsulated photothermal reagents and adjuvant simultaneously into macrophages to generate GNR-PEI/CpG-laden-macrophages for tumor targeting therapy. This strategy provides a potential nanoplatform for tumor therapy by combination of targeted therapy, cell therapy, photothermal therapy and immunotherapy.

\section{Received 7 February 2018; accepted 3 March 2018;} published online 27 March 2018

1 Chen W, Zheng R, Baade PD, et al. Cancer statistics in China, 2015. CA-A Cancer J Clinicians, 2015, 66: 115-132

2 Xu C, Tian H, Chen X. Recent progress in cationic polymeric gene carriers for cancer therapy. Sci China Chem, 2017, 60: 319-328

3 Chen J, Dong X, Feng T, et al. Charge-conversional zwitterionic copolymer as $\mathrm{pH}$-sensitive shielding system for effective tumor treatment. Acta Biomater, 2015, 26: 45-53

4 Wang G, Song W, Shen N, et al. Curcumin-encapsulated polymeric nanoparticles for metastatic osteosarcoma cells treatment. Sci China Mater, 2017, 60: 995-1007

5 Lin L, Guo Z, Chen J, et al. Synthesis and characterization of polyphenylalanine grafted low molecular weight PEI as efficient gene carriers. Acta Polym Sin, 2017, 2: 321-328

6 Xia J, Tian H, Chen J, et al. Polyglutamic acid based polyanionic shielding system for polycationic gene carriers. Chin J Polym Sci, 2016, 34: 316-323

7 Sun W, Gu Z. Tailoring non-viral delivery vehicles for transporting genome-editing tools. Sci China Mater, 2017, 60: 511-515

8 Chen J, Liang H, Lin L, et al. Gold-nanorods-based gene carriers with the capability of photoacoustic imaging and photothermal therapy. ACS Appl Mater Interfaces, 2016, 8: 31558-31566

9 Zhu H, Chen Y, Yan FJ, et al. Polysarcosine brush stabilized gold nanorods for in vivo near-infrared photothermal tumor therapy. Acta Biomater, 2017, 50: 534-545

10 Ye Y, Wang C, Zhang X, et al. A melanin-mediated cancer immunotherapy patch. Sci Immunol, 2017, 2: eaan5692

11 Niu Y, Song W, Zhang D, et al. Functional computer-to-plate nearinfrared absorbers as highly efficient photoacoustic dyes. Acta Biomater, 2016, 43: 262-268

12 Virani NA, Davis C, McKernan P, et al. Phosphatidylserine targeted single-walled carbon nanotubes for photothermal ablation of bladder cancer. Nanotechnology, 2018, 29: 035101

13 Mathiyazhakan M, Upputuri PK, Sivasubramanian K, et al. In situ synthesis of gold nanostars within liposomes for controlled drug release and photoacoustic imaging. Sci China Mater, 2016, 59: 892-
900

14 Yang J, Yao MH, Du MS, et al. A near-infrared light-controlled system for reversible presentation of bioactive ligands using polypeptide-engineered functionalized gold nanorods. Chem Commun, 2015, 51: 2569-2572

15 Liu Y, Yang M, Zhang J, et al. Human induced pluripotent stem cells for tumor targeted delivery of gold nanorods and enhanced photothermal therapy. ACS Nano, 2016, 10: 2375-2385

16 Choi WI, Kim JY, Kang C, et al. Tumor regression in vivo by photothermal therapy based on gold-nanorod-loaded, functional nanocarriers. ACS Nano, 2011, 5: 1995-2003

17 Luo GF, Chen WH, Lei Q, et al. A triple-collaborative strategy for high-performance tumor therapy by multifunctional mesoporous silica-coated gold nanorods. Adv Funct Mater, 2016, 26: 43394350

18 Wang BK, Yu XF, Wang JH, et al. Gold-nanorods-siRNA nanoplex for improved photothermal therapy by gene silencing. Biomaterials, 2016, 78: 27-39

19 Wang F, Shen Y, Zhang W, et al. Efficient, dual-stimuli responsive cytosolic gene delivery using a RGD modified disulfide-linked polyethylenimine functionalized gold nanorod. J Control Release, 2014, 196: 37-51

20 Chen J, Jiao Z, Lin L, et al. Polylysine-modified polyethylenimines as siRNA carriers for effective tumor treatment. Chin J Polym Sci, 2015, 33: 830-837

21 Chen Z, Zhao P, Luo Z, et al. Cancer cell membrane-biomimetic nanoparticles for homologous-targeting dual-modal imaging and photothermal therapy. ACS Nano, 2016, 10: 10049-10057

22 Chen J, Guo Z, Tian H, et al. Production and clinical development of nanoparticles for gene delivery. Mol Ther - Methods Clinical Dev, 2016, 3: 16023

23 Guan $\mathrm{X}$, Guo Z, Lin L, et al. Ultrasensitive $\mathrm{pH}$ triggered charge/size dual-rebound gene delivery system. Nano Lett, 2016, 16: 68236831

24 Guo Z, Chen J, Lin L, et al. $\mathrm{pH}$ triggered size increasing gene carrier for efficient tumor accumulation and excellent antitumor effect. ACS Appl Mater Interfaces, 2017, 9: 15297-15306

25 Cheng Y, Dai Q, Morshed RA, et al. Blood-brain barrier permeable gold nanoparticles: an efficient delivery platform for enhanced malignant glioma therapy and imaging. Small, 2014, 359

26 Li Z, Shao J, Luo Q, et al. Cell-borne 2D nanomaterials for efficient cancer targeting and photothermal therapy. Biomaterials, 2017, 133: $37-48$

27 Patel SK, Janjic JM. Macrophage targeted theranostics as personalized nanomedicine strategies for inflammatory diseases. Theranostics, 2015, 5: 150-172

28 Qian BZ, Pollard JW. Macrophage diversity enhances tumor progression and metastasis. Cell, 2010, 141: 39-51

29 Cao H, Dan Z, He X, et al. Liposomes coated with isolated macrophage membrane can target lung metastasis of breast cancer. ACS Nano, 2016, 10: 7738-7748

30 Choi MR, Stanton-Maxey KJ, Stanley JK, et al. A cellular trojan horse for delivery of therapeutic nanoparticles into tumors. Nano Lett, 2007, 7: 3759-3765

31 Li Z, Huang H, Tang S, et al. Small gold nanorods laden macrophages for enhanced tumor coverage in photothermal therapy. Biomaterials, 2016, 74: 144-154

32 Xie YQ, Wei L, Tang L. Immunoengineering with biomaterials for enhanced cancer immunotherapy. WIREs Nanomed Nanobiotechnol, 2018, 28: e1506 
33 Liu L, Guo Z, Xu L, et al. Facile purification of colloidal NIRresponsive gold nanorods using ions assisted self-assembly. Nanoscale Res Lett, 2011, 6: 143

34 Lohse SE, Murphy CJ. The quest for shape control: a history of gold nanorod synthesis. Chem Mater, 2013, 25: 1250-1261

35 Deng X, Li K, Cai X, et al. A hollow-structured CuS@Cu $\mathrm{Cu}_{2} \mathrm{~S} \mathrm{Au}$ nanohybrid: synergistically enhanced photothermal efficiency and photoswitchable targeting effect for cancer theranostics. Adv Mater, 2017, 29: 1701266

36 Shen J, Kim HC, Mu C, et al. Multifunctional gold nanorods for siRNA gene silencing and photothermal therapy. Adv Healthcare Mater, 2014, 3: 1629-1637

37 Guo L, Yan DD, Yang D, et al. Combinatorial photothermal and immuno cancer therapy using chitosan-coated hollow copper sulfide nanoparticles. ACS Nano, 2014, 8: 5670-5681
Acknowledgements This work was financially supported by the National Natural Science Foundation of China (51390484, 21474104, 51403205, 51503200 and 51520105004), National program for support of Top-notch young professionals, and Jilin province science and technology development program (20160204032GX, 20180414027GH).

Author contributions Chen J, Yan N and Guo Z designed and synthesized the samples; Chen J, Lin L, Hu Y, Fang H and Sun P performed the experiments; Chen J wrote the paper with support from Tian $\mathrm{H}$ and Chen X. All authors contributed to the general discussion.

Conflict of interest The authors declare no conflict of interest.

Supplementary information online version of the paper.
Supporting data are available in the 

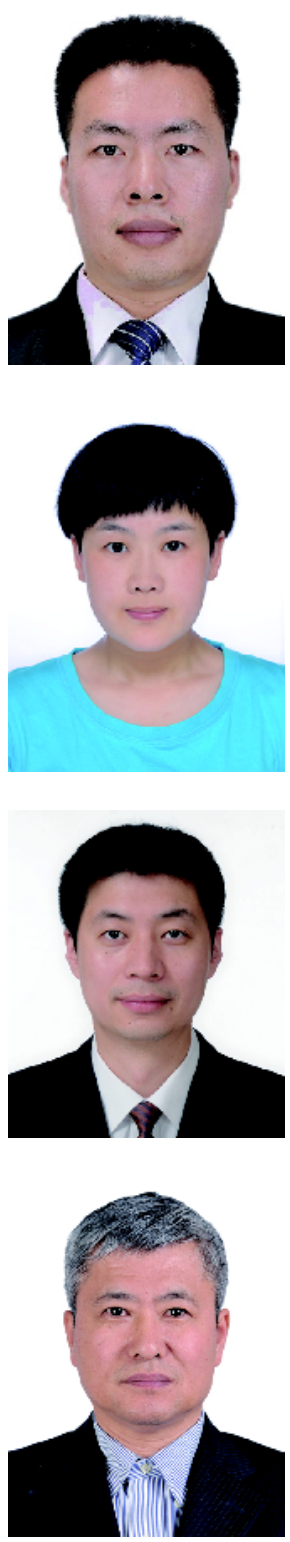

Jie Chen, born in 1982, is currently an associate professor in Changchun Institute of Applied Chemistry, Chinese Academy of Sciences. His research interests are focused on gene delivery and immunotherapy. He has published more than 50 papers in SCI journals and 5 Chinese invention patents.

Lin Lin, born in 1982, is currently an assistant professor in Changchun Institute of Applied Chemistry, Chinese Academy of Sciences. Her research interests are focused on gene carriers design and evaluation. She has published more than 20 papers in SCI journals and 3 Chinese invention patents.

Huayu Tian was born in 1977. Currently, he is a professor in Changchun Institute of Applied Chemistry, Chinese Academy of Sciences. His research interests are focused on polymeric carriers for gene diagnosis and combinational therapy. He published more than 100 papers in SCI journals, such as Progress in Polymer Science, Nano Letters, Biomaterials and Small. He was authorized 15 Chinese invention patents. He was funded by the National Natural Science Funds for excellent Young Scholar and selected for National Program for support of Top-notch Young Professionals.

Xuesi Chen was born in 1959. Currently, he is a professor in Changchun Institute of Applied Chemistry, Chinese Academy of Sciences. His research interests are focused on polymers chemistry on biomedical polymers, drug/gene controlled released carriers designed by biodegradable polymers, bone repair parts and tissue engineering scaffolds from biodegradable polymers. He published more the 500 papers in SCI journals, such as Progress in Polymer Science, Advanced Materials, Advanced Functional Materials, Advanced Drug Delivery Review, and Nano Letters. He was authorized more than 110 Chinese invention patents.

\section{载GNR-PEI/CpG巨噬细胞用于肿瘤的光热和免疫联合治疗}

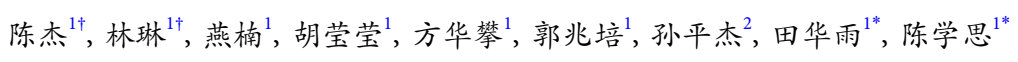

摘要 纳米药物在肿瘤治疗的临床应用是生物医学领域中长期存在的挑战. 主要问题包括: 体内快速清除、脱靶现象以及对转移瘤治疗 的局限性. 本论文用超支化PEI对金纳米棒进行修饰，获得了带有正电性的GNR-PEI，再与负电性的CpG佐剂进行静电复合，形成GNR$\mathrm{PEI} / \mathrm{CpG}$ 纳米复合物. 为了提高体内适用性和靶向性, 我们进一步构建了载GNR-PEI/CpG的巨噬细胞, 用于肿瘤的光热和免疫联合治疗. 体外研究结果表明, 巨噬细胞具有高效的担载GNR-PEI/CpG的能力, 且载GNR-PEI/CpG巨噬细胞具有很好的光热转换能力. 体内研究结 果进一步预示了该策略在肿瘤治疗领域的巨大潜力. 